비대면 교양 영어 수업에서의 패들렛(Padlet) 학습 활용과 학습자 인식 연구

\title{
A Study on the Use of Padlet in Non-Face-to-Face Liberal Arts English Classes and University Students' Responses
}

\author{
김 혜 정1 \\ Kim, HyeJeong ${ }^{1}$
}

\begin{abstract}
The aim of this study is to present learning activities using Padlet to facilitate communication and exchange of opinions among learners in large-scale, remote liberal arts classes, and to examine learners' perceptions of and responses to the use of Padlet as a learning tool. Communication and opinion-sharing activities need to be managed efficiently to minimize the difference in quantity and quality of such activities in remote classes compared to faceto-face. Padlet was actively used for learning activities by 104 college students enrolled in this liberal arts course. In order to broaden the scope of understanding of the content, individual opinions, thoughts, and results of group assignments were uploaded to Padlet. Open-ended questionnaires and interviews were completed to analyze learners' responses to the use of Padlet in a liberal arts class. As a result, it was found that learners had relatively high satisfaction with the use of Padlet in class activities. Specific reasons for satisfaction with using Padlet were found to be "ease of use", "interactive sharing", "fun and interest", and "convenience". In the remote educational era, instructors need to actively use tools such as Padlet for communication and sharing and focus on the development and optimal use of these tools.
\end{abstract}

Keywords: Padlet, communication, sharing, non-face-to-face liberal arts English class, animated movie Applicable level: tertiary

\footnotetext{
1 교신저자, 부교수, 국민대 교양대학, 02707, 서울 성북구 정릉로 77

Corresponding author, Associate professor, College of General Education, Kookmin University, 77 Jeongneung-ro, Seongbukgu, Seoul, 02707, Korea (E-mail: naa4907@kookmin.ac.kr)

Received: January 11, 2022

Revised: February 9, 2022

Accepted: February 22, 2022

Copyright: (C) 2022 The Society for Teaching English through Media (STEM)

This is an open access article distributed under the terms of the Creative Commons Attribution Non-Commercial License (http://creativecommons.org/licenses/by-nc/4.0), which permits unrestricted non-commercial use, distribution, and reproduction in any medium, provided the original work is properly cited.
} 


\section{I. 서론}

2019년 말을 기점으로 전 세계로 확산되어 지금도 진행되고 있는 코로나19 바이러스 사태로 인해 교육계는 급격한 패러다임의 변화를 격고 있다. 교수자들은 비대면 수업에 적합한 새로운 프로그램과 조작 기술을 익혀서 학습 정보를 제공하고 대면 수업에 뒤처지지 않는 학습 환경을 제공하기 위해 고심해야 했다. 학습자들 또한 새롭게 변화된 온라인 학습 환경에 적응하고 좀 더 자기 주도적이고 좀 더 자율적인 자신만의 학습 방안을 설립해야 했다.

기존의 대면 수업을 기반으로 만들어진 수업계획서, 수업 활동 및 수업 절차나 다양한 교수 방안들은 온라인 비대면 수업으로 변경된 환경에서 이러한 변화에 맞게 모두 재편될 필요가 있었다. 예를 들면 대면으로 진행된 수업이 녹화 동영상이나 화상 수업으로 이루어지고 화상 수업에서 활용할 수 있는 수업 활동이나 방안이 새롭게 모색될 필요가 있었다.

코로나19 팬데믹으로 인해 많은 교육 현장에서는 비 대면 온라인 수업을 진행하게 되었고 이에 대한 연구가 발빠르게 진행되고 있다. 많은 연구에서는 온라인 수업의 장점으로 접근의 편이성과 장거리 통학의 불필요성을 포함한 수업 참여의 편리성 등이 발견되었고 반면 인터넷 연결의 불안정성이나 교수자와 학생들간 상호작용의 제한이 온라인 수업의 불편한 점으로 나타났다. 온라인 수업의 질을 향상시키기 위해서는 교수자와 학생들간 적극적인 상호작용과 피드백이 제공되어야 한다(Lim, 2021)는 의견에는 교수자라면 누구나가 공감할 것이다.

학습자들은 정상적인 3 월 개강을 맞이하지 못했기 때문에 개학을 했지만 함께 수업을 듣는 동료들의 얼굴도 제대로 알지 못하며 당연히 친분 관계를 형성할 수도 없다. 화상 강의에서 조별 활동을 진행하더라도 대면수업 때의 조별 활동과 같은 느낌이 나지 않는 것은 아마도 비대면 수업에 따른 학습자들간 사회적 실재감이 낮기 때문일 것이다. 따라서 온라인 비대면 수업에서는 이러한 사회적 실재감을 높이기 위해서라도 학습자들간 상호작용과 의견 공유는 고려되어야 한다. 온라인 수업에서는 상호작용의 양과 질이 중요하며 이것이 학습의 결과로 이어지기 때문에 교사의 상호작용 촉진자로서의 역할이 중요하다는 의견은(Kang \& Kim, 2014) 어쩌면 비대면 수업을 준비하는 모든 교수자들이 유념해야 할 사항일 것이다.

2년여의 시간을 거치면서 교수자나 학습자들 모두 새롭게 조정 된 온라인 비대면 학습 환경에 적응해 가고 있는 시점이지만 대면에서 비대면으로의 전환에서 가장 크게 고려되어야 할 사항 중의 하나는 교수자와 학습자간, 그리고 학습자들 서로들 간의 소통과 의견 공유라 판단된다. 학술연구 정보서비스(RISS)에서 '코로나19와 소통'을 검색하면 2022년 2월 기준으로 그 검색 결과가 1,096건이 나오는 건 이러한 시대적 변화를 잘 반영해 준다고 할 수 있다. 대면 수업에서는 토론 활동이나 조별 활동을 한 후 토론의 결과나 조별 활동의 결과물을 발표하거나 미리 준비해온 자료를 파워포인트 슬라이드에 띄워서 다 함께 공유할 수 있었다. 이러한 활동을 통해 서로 다른 조의 의견을 확인할 수 있고 자신들의 생각을 주장하거나 의견 교환을 통해 학습 내용에 대해 좀 더 밀도 있는 이해를 유도할 수 있었다. 수업 형태가 온라인 비대면으로 전환되면서 이러한 소통과 공유의 모습 또한 변화될 필요가 있다. 대면 수업에서 이루어졌던 의사소통과 의견 공유가 비 대면 수업에서도 다른 형식으로 제공되어야하며 대면 수업에서 비대면 수업으로 전환되면서 생길 수 있는 의사소통과 의견공유의 양과 질의 차이가 최소화되도록 고심할 필요가 있다. 이와 더불어 의사소통과 공유를 촉진시킬 수 있는 효율적 교육 도구의 사용은 함께 고려되어야 한다. 온라인 비대면 시대를 맞이해서 테크놀로지의 사용은 필요한 일이 되었다. 사실 오랜 세월 테크놀로지의 발달은 교육과 밀접한 관계를 이어오고 있으며 테크놀로지는 학생들이 지속적 흥미를 가지고 수업에 참여할 수 있도록 동기를 유발할 수 있는 도구로 작동해 왔다고 해도 과언이 아니다. Laird와 Kuh(2005)는 교육적 목표를 위해 IT 기술의 사용과 적극적이고 협력적 학습과 같은 효율적인 학습 참여에는 긍정적인 관계가 있다고 주장한 바 있다.

이를 위해 본 연구에서는 소통과 공유의 가장 대표적인 도구 중 하나인 패들렛(Padlet)을 활용하고자 하며 이를 이용한 학습 활동 방안을 제시하고, 이에 대한 학습자들의 인식과 반응을 분석하고자 한다. 대면 수업에서 발표를 통해 전체 학생들이 의견 공유를 할 수 있었던 활동을 비대면 수업에서는 패들렛을 적극 활용하여 수업 내용에 대한 깊은 이해를 도모하고 대면 수업에서의 의사소통과 의견 공유의 폭을 확대하고자 하였다. 또한 패들렛을 활용한 수업에 대해 학습자들의 생각과 의견을 수렴하여 분석해 봄으로써 비대면 시대 효율적인 학습 도구로서 패들렛의 활용 방안을 모색해 보고자 한다. 


\section{II. 이론적 배경}

패들렛은 원래 월위셔(Wallwisher)라는 이름으로 시작된 시스템으로 '패들렛'이라는 가상의 게시판에 사용자가 원하는 콘텐츠를 포스팅하고 구성할 수 있고 다른 사람과 실시간으로 공유할 수 있는 협업용 웹 플랫폼을 제공한다(“Padlet,” n.d.). 쉽게 말해 패들렛은 디지털 캔버스를 제공하는 웹 사이트이라 정의할 수 있다. 많은 사용자들이 동시간에 동일한 게시판에 텍스트나 다양한 이미지나 영상을 업로드 할 수 있기 때문에 프로젝트를 함께 해야 할 때 유용한 사이트로 알려져 있다. 패들렛은 기본적으로 가상 게시판(virtual bulletin board)을 활용한 커뮤니티 기반의 인터넷 사이트로 사용자들이 특정 주제에 대한 자신의 생각이나 의견을 쉽게 표현할 수 있으며 다른 사람이 내놓은 의견을 읽어보고 그 의견에 댓글을 달 수 있다. 텍스트, 서류, 이미지, 영상이나, 녹음 파일. 링크 주소 등 어떠한 타입의 콘텐츠도 업로드가 가능하기 때문에 동시다발적인 협동 작업을 가능하게 한다. 교실 환경에서 패들렛은 브레인 스토밍, 토론이나 프로젝트 작업과 같은 학습 활동에 매우 효율적으로 사용될 수 있다. 따라서 패들렛은 학습자의 참여를 도모하는데 효율적인 학습 도구가 될 수 있다(Dunbar, 2017; Garnham \& Betts, 2018). 교실에서 학습자들의 참여와 그들의 생각에 대한 공유는 이미 중요한 학습 요소로 간주되어 왔다.

Students should share, rather than withhold, their insights... Students should give evidence of their active and contextual thinking... Students should examine their beliefs and expose them to critical review by others... Students must find and use their voice as emerging professionals. (Jones, 2008, p. 62)

수업 운영에서 고려되어야 할 학습자들의 통찰력 공유가 가능하다는 기능 외에도 패들렛은 무엇보다 가입해야 할 특별 계정이 필요 없으며 사용을 위해 앱이나 특정 프로그램을 다운로드 해야 하는 번거로움도 없다. 이는 온라인 수업에서 시스템의 편리성이 높으면 높을수록 학습자들의 수업 만족도가 높게 나왔다는 연구 결과(Lee, 2018)에 부합되는 패들렛의 또 하나의 장점이라 할 수 있다.

학생들은 이메일이나 블로그에 URL 을 올리는 것만으로 같은 내용을 공유할 수 있으며 이러한 심플한 접근성이 바로 패들렛이 지니는 가장 큰 장점 중 하나일 것이다. 이러한 특성으로 인해 패들렛은 혁신적인 교육 자원으로 각광받았고 온라인 비대면 수업이 일반화되면서 이에 대한 학문적 연구는 매우 활발히 진행되고 있다(DeWitt \& Koh, 2020; Fisher, 2017; Gill-Simmen, 2021; Goh \& Sandars, 2016; Hamid, Rosli, \& Yunus, 2019; Wood, 2016).

패들렛은 사용자가 공유하기를 원하는 모든 콘텐츠를 포스팅할 수 있는 다양한 게시판을 만들어 낼 수 있는 공간이다. 패들렛은 비어있는 판(blank slate)이기 때문에 사용자가 원하는 콘텐츠로 판을 구성할 수 있고 다른 사람을 초청해서 협업할 수 있다는 특징이 있으며 상호작용할 수 있는 공간이 어떠한 기기로든 어떠한 웹 브라우저든 쉽게 접근할 수 있고 이용하기도 쉽기 때문에 교사와 학생들 모두에 게 유용한 교육용 도구가 될 수 있다(Edward, 2020). 따라서 초기 기존의 많은 연구들은 협업 활동을 지원하기 위한 패들렛의 유용성에 집중된 바 있다(Beltrán-Martín, 2019; Dembo \& Bellow, 2013; Ellis, 2015; Fuchs, 2014; Megat, Yusoff, Azmi, Shariff, \& Hassan, 2020; Rashid, Yunus, \& Wahi, 2019; Sætra, 2021; Zhi \& Su, 2016).

$\operatorname{Sætra}(2021)$ 는 노르웨이 대학원생들을 대상으로 한 연구에서 교실 밖에서의 활발한 학습 활동을 수행할 수 있도록 하기 위해 익명으로 참여하는 패들렛 사용의 효과를 조사하였다. 교수자는 패들렛에 수업 시간에 다루지 않은 여분의 문제를 제출하거나 학생들이 수업 내용과 관련된 질문을 자유로이 올릴 수 있도록 하였다. 매우 쉬운 문제를 수업 시간에 질문하게 되면 부끄럽거나 놀림받을 까 걱정할 수 있는데 익명으로 질문을 올릴 수 있는 패들렛의 사용으로 학생들은 수업 내용에 관해 매우 간단한 질문부터 심오한 질문까지 다양한 질문들을 쏟아냈다고 한다. 이러한 질문에 교수자 뿐만 아니라 내용을 잘 알고 있는 학생도 답변을 해줌으로써 서로 도움을 주는 분위기가 형성되었다고 한다. 이 연구는 패들렛을 기반으로 한 협업 학습은 패들렛 사용이 없던 전통적 수업 집단에게 효율적인 보완책이 될 수 있다는 결론을 내렸다. 또한 패들렛을 활발하게 사용한 학생들은 그것을 자신들의 학습 과정에 대단히 중요한 학습 도구로 인식한다고 보고하였다.

Chun(2021)은 54명의 1 학년 대학생들을 대상으로 패들렛을 활용한 5 가지 다른 포스팅 활동을 수행하고 멀티미디어 학습 게시판(multimedia bulletin board)의 학습 효율성과 이에 대한 학습자들의 인식을 조사하였다. 구체적으로 이 연구는 멀티미디어를 이용한 다양한 온라인 게시판 활동을 통해 학습자들이 이러한 유형의 온라인 도구인 패들렛의 학습 효과를 어떻게 인지하는지를 조사하였다.

패들렛을 이용한 수업 활동의 학습 효과를 정의적, 인지적, 사회적 영역이라는 세 가지 범주로 분류하여 분석하였다. 연구 결과는 세 가지 범주 중 사회적 영역, 정의적 영역순으로 그 학습 효과가 높게 나타났으며 
언어 능력의 증진과 관련된 인지적 영역이 가장 낮게 나온 것으로 보고하고 있다. 학습자들이 멀티미디어를 활용한 포스팅 활동이 자신들의 학습에 대한 동기 유발을 증진시켰다는 응답은 패들렛 활용이 지니는 실질적인 학습 효과라 할 수 있겠다.

패들렛의 활용은 초반 연구가 집중되었던 작문 학습부터(Diab, 2019; Lestari, 2017; Taufikurohman, 2018) 최근에는 말하기 학습까지(Syahrizal \& Rahayu, 2020) 다양한 학습 분야로 세분화되어 연구가 활발히 진행되고 있다. Harris, Yunus, 그리고 Badusah(2017)는 다모드 생산 도구(multimodal production tool)로 패들렛을 규정하고 교수와 학습을 지원하는데 심플하지만 강력한 도구라 정의한 바 있다. 이들은 영어 문법 영역에서 학생들의 학습을 증진시키는데 패들렛 사용의 효율성을 연구하였다. 30 명의 학생들을 대상으로 한 사전-사후 시험과 설문 조사를 통해 패들렛 사용으로 학습자들의 문법 수행에 유의미한 증진이 있었다고 보고하고 있다. 또한 문법을 학습하기 위한 학습 도구로서 패들렛 사용에 대해 학습자들은 긍정적인 태도와 높은 호의를 보였다고 보고하고 있다. 이들은 패들렛의 사용은 언어 학습에서 학생들의 언어 수행을 증진하는데 효율적이라고 주장하였다.

Deni와 Zainal(2018)은 의사소통 기술을 교수하는데 학생들의 학습을 보조하기 위해 사용된 패들렛이 교육적 도구로서 학습에 미치는 영향을 고찰하고자 하였고 이를 위해 교육학적 측면에서 패들렛 사용에 고려해야 할 사항들에 대해 연구하였다. 그들은 의사소통 기술을 교수하는 수업에서 학생들의 학습을 돕기 위한 도구로 패들렛이 사용되기 위해서는 훈련(training), 목적(purpose), 유연성(flexibility), 피드백(feedback), 익명성(anonymity)이 고려되어야 한다고 분석하였다. 학생들은 패들렛 판 작성에 적합한 언어에 대해 훈련을 받을 필요가 있으며 패들렛과 같은 학습 도구가 자신들의 학습을 촉진시키는데 어떻게 사용되어야 하는지 그 사용 목적을 알아야 한다. 또한 학생들이 패들렛에 언제 어디서나 접근할 수 있도록 유연한 접근이 가능해야 한다. 즉 학생들이 쉅게 참여할 수 있도록 하기 위해 과제를 개인별로 낼지 조별로 낼지에 대해서 학생에게 선택권이 주어질 필요가 있으며 과제를 업로드한 후 재수정해서 재제출하는 기한에 제한을 두어서는 안된다는 것이다. 학생들이 패들렛에 올린 과제에 대해 교수자는 피드백을 할 필요가 있으며 이 때의 피드백은 학생 답의 문제점 지적, 어떻게 그것이 더 증진될 수 있는지에 대한 상세한 설명이나 칭찬이 들어가도록 구체적이어야 한다.

Deni와 Zainal(2018)은 마지막으로 패들렛에 학생들의 더 활발한 참여를 독려하기 위해 그룹 이름으로 제출하기와 같은 익명성을 보장할 필요가 있다고 주장하였다. England(2017)는 현대를 살아가는 학생들은 대부분 인터넷과 자연스럽게 연결되어 있고 시각적 자극에 매우 풍족하게 노출되기 때문에 학습에서의 패들렛 활용은 매우 적절하며 특히 $\mathrm{ESL} / \mathrm{EFL}$ 환경에서 패들렛은 매우 자명한 학습 자원이 될 수 있다고 하였다. 또한 패들렛에 포스팅 된 언어 학습 자료의 접근성과 유동성(mobility)은 학생들의 언어 학습을 향상시키고 학습 동기를 부여하는데 도움이 될 수 있다고 하였다.

\section{III. 연구 방법}

\section{1. 연구 대상}

본 연구는 2021년 9월 초부터 12월 초까지 15 주에 걸쳐 진행되었고 서울소재 사립대학교의 교양영어 수업을 듣는 수강생들 104 명을 대상으로 하였다. 1 학년부터 4 학년까지 수강할 수 있는 대규모 교양 강좌이기 때문에 다양한 학과의 학생들로 구성 되었다. 학생들의 전공은 경영 대학 $(52.0 \%, 54$ 명), 법과대학 $(10.6 \%, 11$ 명 $)$, 조형 대학 $(10.6 \%, 11$ 명), 예술대학 $(4.8 \%, 5$ 명 $)$, 과학기술대학 $(3.8 \%, 4$ 명 $)$, 글로벌 인문 지역 대학 $(3.8 \%, 4$ 명), 사회과학대학 $(3.8 \%, 4$ 명 $)$, 자동차융합대학 $(2.9 \%, 3$ 명 $)$, 건축대학 $(1.9 \%, 2$ 명 $), \quad$ 경상대학 $(1.9 \%, 2$ 명 $)$, 체육대학 $(1.9 \%, 2$ 명 $)$, 소프트웨어 융합대학 $(1.0 \%, 1$ 명)과 창의공과대학 $(1.0 \%, 1$ 명 $)$ 이다. 학년 분포도를 살펴보면 1 학년(7.7\%, 8명), 2 학년(38.5\%, 40명), 3학년(38.5\%, 40 명), 4 학년(15.3\%, 16명)으로 구성되었다.

본 연구에서 사용한 교재는 스크린 영어회화 모아나(Screen English: Moana, Kang, 2020)이다. 많은 학습자들이 학습 흥미도적인 면에서 쉅게 접근할 수 있도록 디즈니 애니메이션 모아나(Moana, Clements \& Musker, 2016)를 학습 활동을 위한 자료로 함께 활용하였다. 애니메이션 모아나는 여자 주인공 모아나가 힘든 여정을 겪어 나가는 과정에서 자신의 정체성을 확립해 나가는 성장 스토리라는 측면에서 누구나가 공감할 수 있는 주제이다.

영어 교육을 위한 패들렛 사용 학습에 대해 학습자들이 기본적으로 어떤 의견을 지니고 있는지를 사전 파악하기 위해 수업 첫날 5 점 척도로 구성된 간단한 설문조사를 실시하였다. 전체 학생들 중 95 명이 
응답하였고 “패들렛을 (잘)알고 있거나 들어본 적이 있는가?” 라는 질문에는 “그렇다" 라는 답변에는 $28.4 \%(27$ 명 $)$ 이 응답하였고 “그렇지 않다" $(45.3 \%, 43$ 명)와 “매우 그렇지 않다" $26.3 \%, 25$ 명 $)$ 에는 $71.6 \%$ 가 응답하였다. "영어 수업에서 패들렛을 사용하여 학습해 본 경험이 있는가?" 라는 양자택일형 질문으로(1: 경험이 있다, 2: 경험이 없다)로 구성하였고 “경험이 있다"는 $8.4 \%(8$ 명), “경험이 없다"는 $91.6 \%(87$ 명)이 응답하였다. 본 연구에 참여한 학습자들은 전반적으로 패들렛에 대해 잘 알고 있지 못하며교육 도구로서 패들렛에 대한 사전 경험이 매우 낮은 것으로 나타났다.

\section{2. 연구 절차}

본 연구는 코로나19 바이러스 사태로 인해 학교 방침에 따라 전면 비대면으로 이루어졌으며 기본적으로 수업 녹화 동영상 시청과 화상 수업으로 구성되었다. 수업 녹화 동영상은 매주 가상대학(e-Campus)에 업로드 되었고 화상 수업은 대략적으로 3 주에 한번씩 진행되었다. 전체적으로 한 주의 수업은 수업 전 활동, 수업 활동, 수업 후 활동으로 구성되는데 수업 전 활동은 간단한 퀴즈를 통한 워밍업 활동, 수업 활동은 교재와 관련된 영화 내용 분석 및 표현 학습, 수업 후 활동은 토론 및 조별 활동으로 구성된다. 주로 수업 활동과 수업 후 활동에서 제시된 다양한 학습 활동을 위해 패들렛을 사용하였다.

수업 활동에서는 영화와 관련된 스토리 및 사건을 분석하고 이와 관련된 영어 표현 학습을 중심으로 이루어졌다. 이와 관련된 과제를 개인별로 작성하여 패들렛에 제출하게 한다거나 화상수업에서 조별 활동으로 완성한 과업 결과물을 그룹별로 작성하여 패들렛에 제출하게 하였다. 예를 들어 수업 2 주차에 영화의 도입부에 주인공 모아나의 조력자로 나오는 마우이(Maui)에 대한 소개가 나온다.

Gramma Tala: But in time, some began to seek Te Fiti's heart.... They believed if they could possess it, the great power of creation would be theirs. And one day, the most daring of them all... voyaged across the vast ocean to take it. He was a demigod of the wind and sea. He was a warrior, a trickster, a shape shifter who could change form with the power of his magical fishhook. And his name... was Maui. But without her heart.... Te Fiti began to crumble... giving birth to a terrible darkness.

(대화 1, Moana)

마우이에 대한 소개를 언어 패턴적으로 분석해 보면 $\mathrm{He}$ was $\mathrm{A}, \mathrm{B}, \mathrm{C}$ who 형태로 이루어져 있으며 마우이라는 인물을 전사(warrior), 사기꾼(trickster), 변신할 수 있는 자(shape shifter)로 다양한 측면에서 여러 단어를 사용하여 설명하고 있다. 이러한 언어적 패턴을 활용하여 학습자들 개인별로 각자를 다양한 측면에서 소개해 보도록 하였고 자기 소개서를 패들렛에 업로드 하도록 하였다. 수업 시작 후 2 주가 지난 시점이라 조를 분배하여 조별 활동을 하였다 하더라도 학습자들 간 친밀도는 거의 쌓여 있지 않은 상태이기 때문에 모아나의 할머니 탈라(Tala)가 마우이를 소개하는 장면을 통해 학습자들이 자연스럽게 각자 자기 소개를 해보도록 하였고 이러한 정보를 공유하기 위한 도구로 Figure 1에서처럼 패들렛이 활용되었다.

FIGURE 1

Individual Posting in Padlet

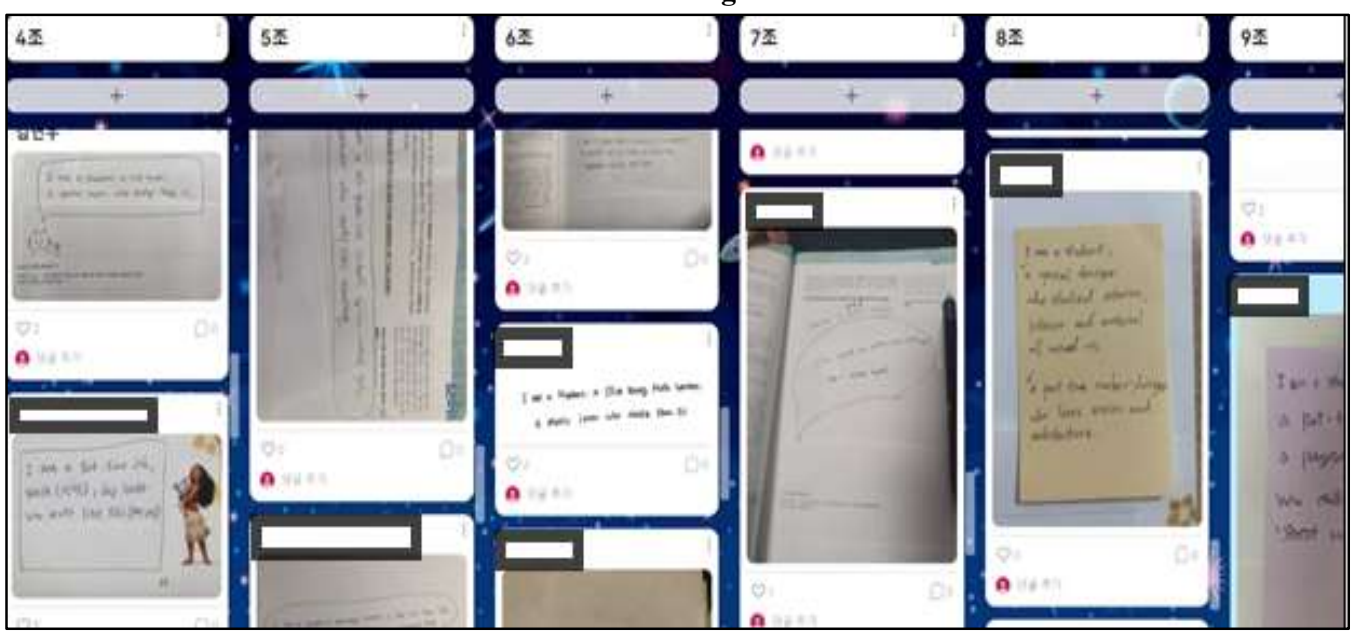


화상 강의에서 전체 학생들이 서로의 얼굴은 볼 수 있다 하더라도 대면 수업에서와 같은 공감대는 형성되기가 쉽지 않으며 줌의 소회의실 기능을 이용하여 조별 활동을 한다 하더라도 자신이 속한 조의 조원들과만 친밀감을 쌓을 수 있다. 이를 해소하기 위해 패들렛 활용을 통한 정보나 의견 공유는 비대면 수업에서 필요한 일이라 판단하였다.

Figure 2는 화상 수업에서 토론 활동이나 조별 활동을 진행했을 경우 조별 토론 활동의 결과나 조별 활동으로 협동하여 작성한 과업 제출물을 패들렛에 공유하도록 한 것이다. 화상 수업 시간내에 업로드하여 학습자들이 실시간으로 다른 조들의 결과물을 함께 볼 수 있도록 하였다. 학습자들에 게 다른 조의 결과물을 살펴보고 “좋아요” 표시를 누르거나 댓글로 자신의 의견을 표현할 수 있도록 하였다.

FIGURE 2

Group Posting in Padlet

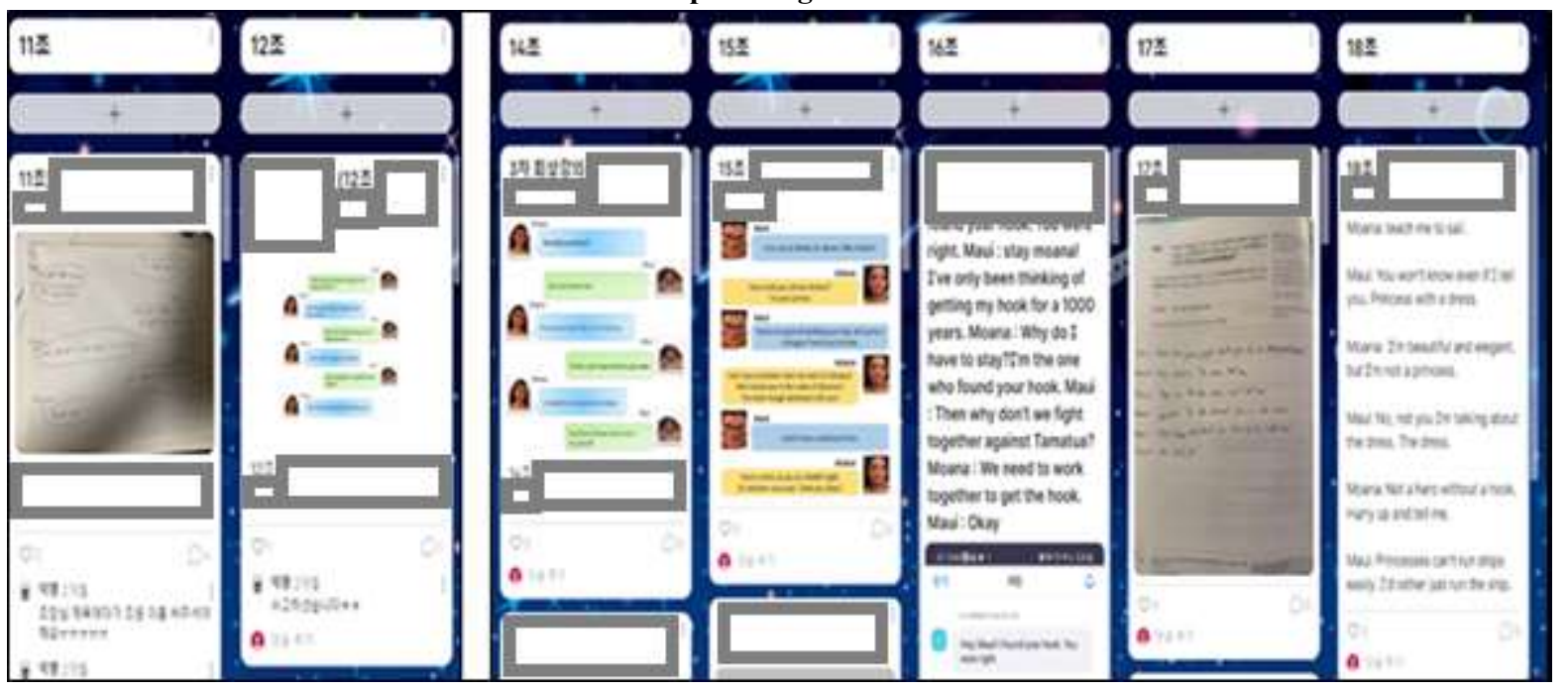

\section{3. 연구 도구}

본 연구의 목적은 교양 영어 수업에서 패들렛을 활용한 학습 활동을 제시하고 이에 대한 학습자 반응과 인식을 파악하고자 하는 것이므로 이를 위한 평가도구로 설문지와 인터뷰를 이용하였다. 설문지는 수업 11 주차에 실시되었고 패들렛 사용에 대한 만족도와 그 이유를 질문하는 두 문항으로 구성되었다. 1 번 문항은 리커트 5점 척도 방식으로 “1. 본 수업에서의 패들렛 활용에 대한 만족도는 어떠한가요?"를 질문하였다(1전혀 만족스럽지 않다, 2-만족스럽지 않다, 3-보통이다, 4-만족스럽다, 5-매우 만족스럽다). 2 번 문항은 개방형 질문 형식으로“2. 위 1번과 같은 답변을 한 이유는 무엇인가요?”를 가감없이 쓰도록 하였다.

인터뷰는 설문 조사만으로 드러나지 않을 수도 있는 학습자들의 개인적인 학습 성향이나 흥미나 관심, 본 연구 목적에 대한 생각이나 태도 등에 대한 심층 자료 수집을 위해 실시되었다. 비대면 수업 방침에 따라 화상 수업이 끝난 후 줌을 활용해 별도로 진행되었으며 자발적으로 참여한 학습자들 48 명을 중심으로 1 인 인터뷰 형식으로 진행되었다. 1 인당 인터뷰 시간은 평균 10-15분 정도 소요되었다. 인터뷰 내용은 학습자들의 동의를 얻어 녹화되었고 인터뷰 내용은 타이핑해서 인터뷰 기록지를 작성하였다.

\section{IV. 연구 결과}

본 연구는 교육 학습 도구로서 패들렛을 활용한 학습 방안을 고찰하고 이에 대한 학습자 반응을 분석하기 위해 패들렛 사용에 대한 만족도를 알아보기 위해 설문조사를 실시하였다. 전체 인원 중 설문조사에 응답한 학습자는 81 명이며 리커트 5점 척도로 질문한 “본 수업에서의 패들렛 활용에 대한 만족도는 어떠한가요?" 라는 문항에 대한 결과를 수치로(\%) 나타내면 다음과 같다. 
FIGURE 3

The Satisfaction on the Use of Padlet

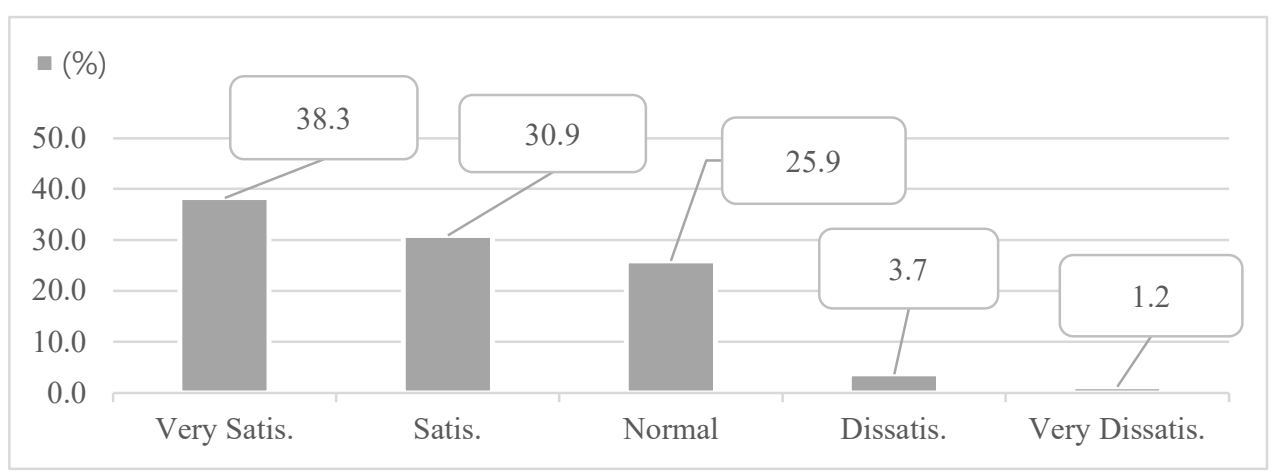

Note. Satis. $=$ Satisfied $/$ Dissatis. $=$ Dissatisfied

Figure 3에서 패들렛 사용에 대한 만족도 결과를 분석해 보면 패들 렛 사용에 '매우 만족한다'는 의견 $(38.3 \%$, 31 명)과 '만족한다'는 의견(30.9\%, 25 명)이 비교적 높게 나타났고 '보통이다'는 의견은 $25.9 \%(21$ 명), '불만족이다'와 '매우 불만족이다'는 의견이 각각 $3.7 \%(3$ 명 $), 1.2 \%(1$ 명)으로 나타났다. 매우 만족한다는 의견과 만족한다는 의견을 통해 전반적으로 학습자들은 본 수업에서의 패들렛 활용에 만족도가 꽤 높은 편인 것으로(69.2\%) 판단할 수 있겠다. 이에 대한 구체적인 이유를 개방형 형식으로 질문하였고 학습자들은 다양한 이유를 제시하였다. 학습자들의 의견을 체계적으로 정리하기 위해 만족도나 불만족도에 대한 이유를 의미단위로 코딩하여 범주화 하였다. 먼저 패들렛 사용에 대해 만족한다는 긍정적인 의견에 대한 이유는 패들렛 사용의 용이성, 상호작용적 공유, 재미와 흥미, 편리성, 기타 항목으로 범주화 되며 학습자들의 구체적인 의견은 Table 1과 같다.

TABLE 1

The Reasons of Satisfaction on the Use of Padlet

\begin{tabular}{|c|c|c|}
\hline Category & $N(\%)$ & Reasons \\
\hline Easy to Use & $\begin{array}{c}21 \\
(25.9 \%)\end{array}$ & $\begin{array}{l}\text {-It is easy to use and fast. } \\
\text {-It is not difficult to use as the site is similar to the program I normally use. } \\
\text {-I first learned about Padlet while taking this class, but it's easy to upload files and it's good. } \\
\text {-Uploading is simple and there is no need to worry about server errors. }\end{array}$ \\
\hline $\begin{array}{l}\text { Interactive } \\
\text { Sharing }\end{array}$ & $\begin{array}{c}32 \\
(39.5 \%)\end{array}$ & $\begin{array}{l}\text {-It was nice to be able to see the tasks of other members in real time. } \\
\text {-We can share assignments with everyone so we can compare how others did it. } \\
\text {-It is nice to be able to share the tasks of the members on various tasks. } \\
\text {-I think it's a useful site for seeing the activities of various people and being able to share them } \\
\text { with each other. } \\
\text {-It is very satisfying to be able to see the activities of other members and give feedback. } \\
\text {-It was nice to be able to see all the results of the other groups and to comment and exchange } \\
\text { opinions with each other. } \\
\text {-Most of all, it was nice to be able to express my opinions interactively and comfortably through } \\
\text { comments. }\end{array}$ \\
\hline Fun \& Interest & $\begin{array}{c}5 \\
(6.2 \%)\end{array}$ & $\begin{array}{l}\text {-It's fun to skim through the other groups' assignments. } \\
\text {-It was fun because it was my first time using the tool. } \\
\text {-I was intrigued by the task because I was using it for the first time. }\end{array}$ \\
\hline Convenience & $\begin{array}{c}10 \\
(12.3 \%)\end{array}$ & $\begin{array}{l}\text {-I am so glad I learned this Padlet. It seems convenient to upload group assignments. } \\
\text {-I was able to use the Padlet to increase participation in class and to use it conveniently for } \\
\text { class activities. } \\
\text {-The process is not complicated and it is very convenient to submit assignments. } \\
\text {-There were no errors and it was convenient to access not only from PC but also mobile. }\end{array}$ \\
\hline Etc. & $\begin{array}{c}4 \\
(5 \%)\end{array}$ & $\begin{array}{l}\text {-It is a new and fresh tool. } \\
\text {-I've heard of Padlets before, and it's amazing to try it out myself. } \\
\text {-It was wonderful to know that such an educational site. }\end{array}$ \\
\hline
\end{tabular}

패들렛 사용에 대해 만족하는 이유로 $25.9 \%$ 의 학생들이 패들렛 사용의 용이성이라고 응답하였다. 접근성이 쉽고 누구나가 쉽게 사용할 수 있는 패들렛의 장점을 학습자들이 인지한 것으로 판단된다. 본인이 사용하는 프로그램이랑 유사해서 사용하는데 어려움이 없었다거나 이 수업을 통해 패들렛을 처음 알게 되었지만 파일을 업로드 하는게 쉬웠다는 의견, 업로드가 간단하고 서버 오류에 대한 걱정이 없다는 의견 
등은 새로운 학습 도구로서 제공된 패들렛에 대해 쉽게 접근해서 어렵지 않게 사용할 수 있다는 점에 학습자들이 만족하는 것으로 나타났다.

다음으로 패들렛 사용에 만족하는 이유로 가장 높게 나타난 이유는(39.5\%) 상호작용적 공유이다. 다른 사람들의 과업 내용을 모두 함께 공유할 수 있다는 것이 패들렛 사용의 가장 큰 장점이며 이 장점의 극대화가 효율적으로 적용되었음을 알 수 있다. 실시간으로 다른 조의 과업을 볼 수 있어서 좋았다는 의견, 다양한 과업 내용을 공유할 수 있어서 좋다는 의견, 다양한 사람들의 학습 활동을 훓어보는데 유용하다는 의견 등은 공유라는 개념 자체가 실현화 된 것에 대한 만족으로 파악된다. 또한 댓글로 의견을 교환할 수 있어서 좋다는 의견이나 댓글 기능을 통해 자신의 의견을 편하게 표현하고 그 과업을 한 조원들의 의견을 듣고 하는 상호작용적 기능에 대한 만족감이 함께 나타났다. 이러한 상호작용적 공유에 대한 만족감은 인터뷰에서도 분명하게 나타났다.

가상대학 과제란에 과제를 올리면 저희는 다른 조는 어떻게 했는지 알 수가 없잖아요. 교수님만 보시는 거고... (중략) 우리 조가 한 게 제대로 한 건지 다른 조는 어떻게 했는지 좀 궁금할 때도 있거든요. 그래서 패들렛으로 전체 조가 한 것을 홇어보는 건 진짜 너무 신선했어요. (법과대학, 2학년, Kim, S. J.)

사실 다른 조가 올린 과제를 보는 건 우리 조가 잘 했는지 상대적 비교가 돼서 우리 조의 과제 수준을 가늠할 수 있어서 좋은 거 같아요. 그리고 과제만 올리고 그냥 끝나는 게 아니라 다른 조 과제 댓글란에 질문을 하면 답변이 바로 달리고 하는 거 보면 이게 진정한 상호작용이고 대학 수준다운 수업이지 않나.... (중략) (경영 대학, 4 학년, Shin, K. S.)

이렇듯 공유만 하고 그치는 것이 아니라 공유한 과제물에 대해 서로의 의견을 교환할 수 있고 그러한 의견 교환 또한 공유된다는 점에 학습자들은 만족감을 보이는 것으로 나타났다. 다음으로 학습자들이 패들렛 사용에 만족하는 이유로 나온 의견은 재미와 흥미 $(6.2 \%)$ 이다. 다른 조의 과제물을 훓어보는 일이 재미있었다거나 처음 이 사이트를 이용해 보는데 재미있었다는 의견, 그리고 처음 사용해 보기 때문에 업로드 된 과제에 흥미가 생겼다는 의견 등을 통해 패들렛 사용을 재미있는 활동으로 인식하는 학습자들이 있다는 것을 알 수 있다. 마지막으로 패들렛 사용에 만족하는 이유로 편리성 $(12.3 \%)$ 이라는 의견이 나왔다. 편리성은 첫번째 범주로 고찰해 본 사용의 용이성과는 다소 다른 개념이라 하나의 범주로 분류하였다. 과제를 업로드하는데 편리했다거나 교실 활동에 참여하는 데 편리하다는 의견, $\mathrm{PC}$ 뿐만 아니라 모바일로도 접근하기 편했다는 의견 등은 교육도구로서 패들렛의 사용이 편리하다는 것으로 판단된다. 패들렛 사용의 편리성에 대한 의견은 인터뷰에서도 나타났다.

...(중략) 이런 종류의 사이트가 다 그런 거 같아요. 저는 모바일로 수업도 듣고 화상 강의도 참여하고 거의 모든 걸 모바일로 하는데 녹화 영상 같은 경우는 모바일로 시청하면 기록 안되는 경우도 있는데.... (중략)... 패들렛은 언제 어디서나 모바일로 편하게 들어가서 볼 수 있고 지난번에 올린 과제도 누적되어 있어서 다시 보기에도 편리하고 ...(중략) 저는 이 수업에서 패들렛을 처음 사용하는 건 데요, 개인적으로 패들렛이 편리한 거 같아요. (중어중문, 1 학년, Lee, D. M.)

다음으로 패들렛 사용에 대한 불만족스러운 점과 개선 점에 대해 분석해 보고자 한다. 패들렛 사용에 대해 만족하지 못하는 이유는 익숙치 않음으로 나타났고 구체적인 의견은 Table 2와 같다.

패들렛 사용에 만족하지 못하는 이유로는 처음 사용하는 사이트라 낯설고 생소했다거나 익숙치 않은 작업이라는 의견이 $6.2 \%$ 나왔다. 본 수업을 통해 패들렛을 처음 사용해봤고 그래서 패들렛 사용이 익숙치 않았다거나 생소해서 사용하기 불편했다는 의견, 익숙한 가상대학에 과제를 제출하는 것이 훨씬 쉽다고 한 의견은 교육 도구나 사이트의 익숙함이 학습자들에게 또 하나의 변수가 될 수 있음을 알 수 있게 한다. 이는 곧 패들렛을 처음 접해본 학습자들을 위해 고려해야 할 사항을 파악할 수 있게 하는 의견들이다. 학습자에 따라 교육 도구의 익숙함이나 친숙함이 중요하게 작용할 수도 있기 때문에 패들렛을 처음 접하는 학습자들에게는 이에 익숙해질 수 있도록 간단한 연습용 과업을 제출하게 하거나 반복되는 충분한 설명을 통해 쉽게 접근할 수 있도록 할 필요가 있겠다. 
TABLE 2

The Reasons of Dissatisfaction on the Use of Padlet and Improvements

\begin{tabular}{|c|c|c|}
\hline Category & $N(\%)$ & Reasons \\
\hline $\begin{array}{l}\text { Unaccustomed } \\
\text { work }\end{array}$ & $\begin{array}{c}5 \\
(6.2 \%)\end{array}$ & $\begin{array}{l}\text {-It's the first site I've used, so I'm not familiar with it yet. } \\
\text {-Padlet has good accessibility, but it is difficult to edit uploaded data. } \\
\text {-Since each member participates on a PC or mobile device, uploading files to the Padlet in } \\
\text { groups was not smooth. } \\
\text {-Submitting assignments in the familiar virtual campus is much easier. } \\
\text {-It's a site I'm not familiar with, so it's inconvenient to use. }\end{array}$ \\
\hline Improvements & $\begin{array}{c}3 \\
(3.7 \%)\end{array}$ & $\begin{array}{l}\text {-If the professor upload the Padlet site address to the virtual campus, it will be more accessible. } \\
\text {-If it is mandatory to comment on the assignments of other groups, it seems to create a more } \\
\text { lively discussion. }\end{array}$ \\
\hline Etc. & $\begin{array}{c}1 \\
(1.2 \%)\end{array}$ & $\begin{array}{l}\text {-I felt it would be more helpful to upload each individual work on Padlet. I think it is important } \\
\text { to coordinate the opinions of the members in group activities... I felt that there were restrictions } \\
\text { on the activities in video lectures using zoom. }\end{array}$ \\
\hline
\end{tabular}

개선 사항으로는 패들렛 사이트 주소를 가상 대학 공지란에 고정된 정보로 올려 달라는 의견이 있었다. 수업 시간마다 패들렛 주소를 매번 별도로 공지해서 제공했는데 사이트 주소를 언제든 열람할 수 있게 공지란에 고정된 정보로 제공할 필요가 있 겠다. 또한 다른 조의 과제물에 대해 좋아요 표시를 누르거나 댓글 달기와 같은 부가적인 기능은 학습자들의 자율에 맡겼는데 이를 의무적으로 하도록 유도하면 좀 더 생생한 토론 느낌이 날 것 같다는 의견도 있었다. 수업에 패들렛 활용시 댓글 달기 활동을 학습자들의 자율에 맡길지 모두가 해야 하는 의무적 사항으로 할지는 수업을 수강하는 학습자들과의 의견을 수렴하여 정해서 공지할 필요가 있겠다.

\section{V. 결론 및 제언}

본 연구는 비대면으로 진행된 교양영어 대규모 강좌에서 학습자들 간의 소통과 의견 교환 및 수업 활동 공유를 원활히 하기 위해 패들렛 학습 활용 방안을 고찰하고 이에 대한 학습자 인식과 반응을 연구하고자 하였다. 기존의 대면 수업에서 자연스럽게 이루어지던 의사소통과 의견 공유 활동을 온라인 비대면 수업에서도 그러한 활동의 양과 질에 있어서의 차이를 최소화하여 효율적으로 진행될 필요가 있다.

이를 위해 교양영어를 수강한 대학생 104명을 대상으로 비대면으로 진행된 다양한 수업 활동에서 패들렛을 적극 활용하였다. 수업 내용에 대한 분석과 이해의 폭을 넓히기 위해 학습자들에게 개인의 의견이나 생각을, 또는 조별 과제를 통한 결과물 등을 패들렛에 업로드 하도록 하였다. 패들렛에 올라온 다른 학습자나 다른 조의 결과물을 탐색하고 “좋아요” 표시를 누르거나 댓글을 자유로이 달도록 유도하였다. 개별적 포스팅과 조별 포스팅을 통해 수업 활동에 패들렛을 활발히 활용하고자 하였다.

이러한 수업 활동 후 교양 영어 수업에서 패들렛 활용에 대한 학습자들의 반응을 분석하기 위해 설문조사와 인터뷰를 실시하였다. 그 결과 학습자들은 수업 활동에 활용된 패들렛 사용에 대해 만족도가 비교적 긍정적인 것으로 나타났다(69.2\%). 패들렛 활용에 대해 만족하는 구체적인 이유로는 패들렛 사용의 용이성, 상호작용적 공유, 재미와 흥미, 편리성으로 나타났다. 다른 사람이나 다른 조의 과업 내용을 실시간으로 공유할 수 있어서 좋았다거나 다양한 학습자들의 학습 활동을 훝어보는 데 유용했다거나, 댓글로 의견 교환할 수 있어서 만족스러웠다는 상호작용적 공유와 관련된 의견이 패들렛 활용의 만족 이유 중 수치가 가장 높게 나타났다. 패들렛을 수업에 활용하고자 하는 교수자들은 이것이 패들렛 사용의 가장 큰 장점임을 인지하여 이러한 장점을 극대화하기 위한 좀 더 흥미롭고 다양한 학습 방안에 대해 고심해 볼 필요가 있겠다.

패들렛 사용에 만족하지 못하는 이유로는 익숙치 않거나 다소 낯설고 생소한 활동이라는 의견이 나왔다. 패들렛을 본 수업에서 처음 접해본 학습자들은 기존에 사용하던 가상대학 과제 제출란을 오히려 편안하게 생각하였다. 이를 통해 아무리 학습에 효율적인 학습 도구라 하더라도 학습자들이 익숙치 않으면 불편하게 생각할 수 있다는 것을 파악할 수 있다. 이러한 의견을 바탕으로 교수자들은 패들렛에 처음 접하는 학습자들을 위해 상세한 지침 설명을 반복적으로 제공한다거나 간단한 연습용 과업 제출을 통해 정해진 기간 내에 패들렛 사용에 쉽게 익숙해질 수 있도록 보완할 필요가 있겠다. 차후 패들렛을 활용한 세분화된 학문 분야별 교수 방안이나 패들렛이 실질적인 학습의 효율성에 미치는 영향에 관한 후속 연구가 활발히 진행되어야 할 것이다. 
본 연구가 대규모 교양 강좌라고 해도 특정 학교의 특정 집 단을 대상으로 한 점, 다양한 패들렛 활용 기능 중 특정 기능만을 사용한 점, 패들렛 활용을 통한 학습에 미치는 효과 등에 대한 양적 평가가 결여된 점은 연구의 한계점이라 할 수 있다. 하지만 온라인 비대면 시대 새롭게 변화된 학습 환경에 맞춰 학습자들과의 소통과 의견 공유의 방식 또한 변화될 필요가 있으며 이를 위한 하나의 학습 도구로서 패들렛의 활용과 이에 대한 학습자 반응을 고찰하였다는 점에서 연구의 의의를 찾을 수 있겠다.

본 연구 결과를 바탕으로 다음과 같은 제언을 하고자 한다. 첫째, 대면수업에서 자연스럽게 이루어지던 소통과 결과물 공유가 온라인 비대면 수업에서도 동일하게 이루어질 필요가 있다. 기존의 대면 수업을 기준으로 형성되어 있던 수업 계획, 수업 활동 및 수업 평가 등의 모든 항목들은 온라인 비대면 수업으로 전환됨에 따라 전면 수정 될 필요가 있다. 이를 위한 하나의 일환으로 패들렛과 같은 다양한 교육적 도구의 사용이 더욱 활발히 시도되고 연구되어져야 할 것이다.

둘째, 대면 수업과 달라진 온라인 비대면 수업 환경에서 학습자들은 강의 콘텐츠의 질적인 부분 뿐만 아니라 수업 시간 내 이루어지는 상호작용과 소통도 중요시 한다는 것을 인지할 필요가 있다. 이를 위해 본 연구에서는 패들렛의 사용과 그 가치를 고찰하였지만 교수자와의 소통 및 학습자 간의 편안하고 부담 없는 소통과 공유가 이루어질 수 있도록 다양한 시도가 이루어져야 할 것이다.

셋째, 변화하는 시대에 맞춰 교수자들의 역할 또한 변모되어야 할 것이다. 학습자들 뿐만 아니라 교수자들 또한 코로나19 팬 데믹으로 인해 온라인 비 대면 수업으로의 갑작스럽고도 빠른 변화를 경험 했다. 교수자들은 교육적 패러다임의 빠른 변화에 맞춰 가기 위해 동영상을 녹화하고 편집하는 기술부터 실시간 원격 수업을 위한 줌(Zoom), 행아웃(Hangout), 팀즈(Teams)와 같은 다양한 화상 수업 도구의 사용법까지 익혀야 했다. 교수자들의 이러한 노력은 지속되어야 할 것이며 시대적 변화에 빠르게 적응하고 앞서 나가기 위해 디지털 리터러시(digital literacy) 능력의 함양을 위해 정진해야 할 것이다.

\section{REFERENCES}

Beltrán-Martín, I. (2019, June). Using Padlet for collaborative learning. Paper presented at the 5th International Conference on Higher Education Advances, Valencia, Spain.

Chun, S. I. (2021). College students' perception of the learning effectiveness of Padlet. Studies in English Education, 26(3), 485-506. https://doi.org/10.22275/SEE.26.3.05

Clements, R., \& Musker, J. (Directors). (2016). Moana [Motion picture]. United States: Disney Animation Studio.

Dembo, S. E., \& Bellow, A. S. (2013). Untangling the web: 20 tools to power up your teaching. London: SAGE. https://doi.org/10.4135/9781506374642

Deni, A., \& Zainal, I. Z. (2018, December). Padlet as an educational tool: Pedagogical considerations and lessons learnt. Paper presented at the10th International Conference on Education Technology and Computers, Tokyo, Japan.

DeWitt, D., \& Koh, E. H. (2020). Promoting knowledge management processes through an interactive virtual wall in a postgraduate business finance course. Journal of Education for Business, 95(4), 255-262. https://doi.org/10.1080/08832323.2019.1635977

Diab, A. (2019). Using some online-collaborative learning tools (Google Docs \& Padlet) to develop student teachers' EFL creative writing skills and writing self-efficacy. Journal of Faculty of Education, 30, 21-70. https://doi.org/10.21608/jfeb.2019.61360

Dunbar, L. (2017). Using Padlet to increase student interaction with music concepts. General Music Today, 30(3), 26-29. https://doi.org/10.1177/1048371316687076

Edwards, L. (2020, October 19). What is Padlet and how does it work for teachers and students? Tech \& Learning: Tools \& Ideas to Transform Education. Retrieved from https://www.techlearning.com/how-to/what-is-padletand-how-does-it-work-for-teachers-and-students

Ellis, D. (2015). Using Padlet to increase student engagement in lectures. In A. Jefferies \& M. Cubric (Eds.), Proceedings of the $14^{\text {th }}$ European Conference on E-Learning (pp. 195-198). New York, NY: Curran Associates.

England, S. (2017). Tech for the modern EFL student: Collaborate and motivate with Padlet. Accents Asia, 9(2), 5660. Retrieved from http://www.issues.accentsasia.org/issues/9-2/England.pdf 
Fisher, C. D. (2017). Padlet: An online tool for learner engagement and collaboration. Academy of Management Learning and Education, 16(1), 163-165. https://doi.org/10.5465/amle.2017.0055

Fuchs, B. (2014). The writing is on the wall: Using Padlet for whole-class engagement. Library Orientation Exchange Quarterly, 40(4), 7-9. Retrieved from https://uknowledge.uky.edu/libraries_present/80/

Garnham, W. A., \& Betts, T. (2018). The Padlet project: Transforming student engagement in foundation year seminars. Compass: Journal of Learning and Teaching, 11(2). Retrieved from https://journals.gre.ac.uk/index.php/compass/article/view/714/0

Gill-Simmen, L. (2021). Using Padlet in instructional design to promote cognitive engagement: A case study of undergraduate marketing students. Journal of Learning Development in Higher Education, 20, 1-14. https://doi.org/10.47408/jldhe.vi20.575

Goh, P. S., \& Sandars, J. (2016). An innovative approach to digitally flip the classroom by using an online "graffiti wall" with a blog. Medical Teacher, 38(8), 858. https://doi.org/10.1080/0142159X.2016.1204433

Hamid, A. A., Rosli, L. N., \& Yunus, M. M. (2019). Wall attack in Padlet in enhancing vocabulary acquisition. International Journal of Academic Research in Business and Social Sciences, 9(1), 563-572. https://doi.org/10.6007/ijarbss/v9-i1/5458

Harris, M., Yunus, M. M., \& Badusah, H. J. (2017). The effectiveness of using Padlet in ESL classroom. International Journal of Advanced Research, 5(2), 783-788. https://doi.org/10.21474/IJAR01/3214

Jones, R. C. (2008). The "why" of class participation: A question worth asking. College Teaching, 56(1), 59-63. https://doi.org/10.3200/CTCH.56.1.59-64

Kang, Y. H. (2020). Screen English: Moana. Seoul: Gilbut.

Kang, M. S., \& Kim, M. R. (2014). The scale development for instructor's role in online teaching. Journal of Korean Association for Educational Information and Media, 20(4), 521-545. https://doi.org/10.15833/KAFEIAM.20.4.521

Laird, T. F. N., \& Kuh, G. D. (2005). Student experiences with information technology and their relationship to other aspects of student engagement. Research in Higher Education, 46(2), 211-233. https://doi.org/10.1007/s11162-004-1600-y

Lee, S. C., \& Kim, J. A. (2018). Factors that affect student satisfaction with online courses. The Journal of Educational Administration, 36(2), 115-138. https://doi.org/10.22553/keas.2018.36.2.115

Lestari, S. (2017). Implementing Padlet application to improve writing ability in English writing skill for non English department students. Linguistics Literature English Teaching, 7(1), 1-16. Retrieved from http://jurnal.uinantasari.ac.id/index.php/let/article/view/1640/1211

Lim. J. W. (2021). A study of college English education majors' perceptions on online class operations in the midst COVID-19 pandemic. English Language \& Literature Teaching, 27(3), 109-128. https://doi.org/10.35828/etak.2021.27.3.109

Megat, N., Yusoff, R., Azmi, N., Shariff, S., \& Hassan, W. (2020). Enhancing classroom engagement through Padlet as a learning tool: A case study. International Journal of Innovative Computing, 10(1), 49-57. https://doi.org/10.11113/ijic.v10n1.250

Padlet. (n.d.). In Wikipedia. Retrieved December 31, 2021, from https://en.wikipedia.org/wiki/Padlet

Rashid, A. A., Yunus, M. M., \& Wahi, W. (2019). Using Padlet for collaborative writing among ESL learners. Creative Education, 10(3), 610-620. https://doi.org/10.4236/ce.2019.103044

Sætra, S. H. (2021). Using Padlet to enable online collaborative mediation and scaffolding in a statistics course. Education Science, 11, 219-229. https://doi.org/10.3390/educsci11050219

Syahrizal, T., \& Rahayu, S. (2020). Padlet for English speaking activity: A case study of pros and cons on ICT. Indonesian EFL Journal, 6(2), 149-156. https://doi.org/10.25134/ieflj.v6i2.3383

Taufikurohman, S. I. (2018). The effectiveness of using Padlet in teaching writing descriptive text. Journal of Applied $\begin{array}{lllll}\text { Linguistics } \quad \text { Lnd } & \text { 2(2), } & \text { Retrieved }\end{array}$ https://jurnal.unigal.ac.id/index.php/jall/article/view/2190/2398

Wood, M. (2016). Padlet: A graffiti wall for today's agriculture teacher. The Agricultural Education Magazine, 88(6), $20-22$. 
Zhi, Q., \& Su, M. (2016). Enhance collaborative learning by visualizing process of knowledge building with Padlet. In IEEE (Institute Electrical and Electronics Engineers) (Ed.), Proceedings of 2015 International Conference of Educational Innovation Through Technology, EITT 2015, 1 (pp. 221-225). Wuhan, China: IEEE. https://doi.org/10.1109/EITT.2015.54 